\title{
Progress in use of carbon-black- polymer composite vapor detector arrays for land mine detection
}

Shawn M. Briglin, Michael C. Burl, Michael S. Freund, Nathan Saul Lewis, Adam J. Matzger, et al.

Shawn M. Briglin, Michael C. Burl, Michael S. Freund, Nathan Saul Lewis, Adam J. Matzger, D. Nelson Ortiz, Phil Tokumaru, "Progress in use of carbon-black-polymer composite vapor detector arrays for land mine detection," Proc. SPIE 4038, Detection and Remediation Technologies for Mines and Minelike Targets V, (22 August 2000); doi: 10.1117/12.396281 


\title{
Progress in Use of Carbon Black-Polymer Composite Vapor Detector Arrays for Land Mine Detection
}

\author{
Shawn M. Briglin ${ }^{\mathrm{a}}$, Michael C. Burl ${ }^{\mathrm{b}}$, Michael S. Freund ${ }^{\mathrm{c}}$, Nathan S. Lewis* ${ }^{\mathrm{a}}$, Adam Matzger ${ }^{\mathrm{a}}$, \\ D. Nelson Ortiz ${ }^{\mathrm{a}}$, Phil Tokumaru ${ }^{\mathrm{d}}$ \\ ${ }^{a}$ Division of Chemistry and Chemical Engineering, Noyes Laboratory, 127-72 \\ California Institute of Technology, Pasadena, CA 91125 \\ ${ }^{b} J e t$ Propulsion Laboratory, California Institute of Technology, Pasadena, CA 91109 \\ 'Beckman Institute, California Institute of Technology, Pasadena, CA 91125 \\ ${ }^{\mathrm{d}}$ AeroVironment Inc., Monrovia, CA 91016
}

\begin{abstract}
Thin films of carbon black-organic polymer composites have been deposited across two metallic leads, with swelling-induced resistance changes of the films signaling the presence of vapors. To identify and classify vapors, arrays of such vapor sensing elements have been constructed. Each element contained a different organic polymer as the insulating phase. The differing gas-solid partition coefficients for the various polymers of the detector array produced a pattern of resistance changes that was used to classify vapors and vapor mixtures. The performance of this system towards DNT, the predominant signature in the vapor phase above land mines, has been evaluated in detail, with robust detection demonstrated in the laboratory in less than $5 \mathrm{~s}$ in air at DNT levels in the low ppb range.
\end{abstract}

Keywords: Land mine, vapor sensors, DNT, carbon black

\section{INTRODUCTION}

Prior work in our laboratory has utilized broadly responsive detector arrays based on carbon black composites ${ }^{1-3}$ for various vapor detection tasks. In this approach, individual detector elements are constructed from films consisting of carbon black particles dispersed into insulating organic polymers. The carbon black endows electrical conductivity to the films, whereas the different organic polymers are the source of chemical diversity between elements in the detector array. Swelling of the polymer upon exposure to a vapor increases the resistance of the film, thereby providing an extraordinarily simple means for monitoring the presence of a vapor. ${ }^{1,4-10}$ Because different polymer compositions are present on each detector element, an array of elements responds to a wide variety of vapors (or complex mixtures of vapors) in a distinctive, identifiable fashion. The electrical resistance signals that are output from the array can be readily integrated into software- or hardware-based decision systems, allowing for an integration of sensing and analysis functions into a compact, low-power, simple vapor detector.

In the present work, we have focused on the performance of such carbon black-polymer composite detector arrays towards 2,4-dinitrotoluene (DNT). This compound has been reported to comprise the predominant vapor phase signature above land mines, ${ }^{11}$ and the presence of DNT has been associated with the success of canines as well as with the success of novel vapor detection schemes for locating buried mines in the field. ${ }^{12}$ We describe measurements of the sensitivity of a particular representative detector towards DNT as a function of exposure time, flow rate, and DNT concentration. In addition, we describe progress in developing a sampler for efficiently transporting DNT packets in air to the detector films of interest and describe the underlying engineering principles that have guided our sampler design. 


\section{EXPERIMENTAL}

Two separate types of experiments were performed. The procedures for each set are described briefly in this section.

\subsection{Response at Constant Flow Rate to $5 \mathrm{~s}$ Pulses of DNT in Air}

$5 \%$ of the vapor pressure of DNT at $20^{\circ} \mathrm{C}$ was selected as a dilution of DNT that would deliver less than $10 \mathrm{ppb}$ of the compound to the detectors. The DNT source was a tube approximately a meter in length that beld about $180 \mathrm{~g}$ of loosely packed, granulated DNT. The air flow through the tube was $0.5 \mathrm{~L}-\mathrm{min}^{-1}$. This air flow was mixed with, and therefore diluted by a flow of $9.5 \mathrm{~L}-\mathrm{min}^{-1}$ of air (from the same source) that did not flow through the DNT tube. Flows were controlled by mass flow controllers in a computer controlled system that bas been described in detail previously. ${ }^{13}$ A union- $\mathrm{T}$ was used to mix the background and analyte-containing gases, and a short Teflon tube was connected to the output of the union to direct the gas toward the bank of detectors. At this dilution, the upper limit of the DNT concentration is $7 \mathrm{ppb}$, because the vapor pressure of DNT at room temperature is approximately $140 \mathrm{ppb}$. If saturation of the background air through the DNT tube occurred, and if no DNT stuck to the walls of the tubing after mixing with the pure background analyte flow, this dilution would produce a concentration of $7 \mathrm{ppb}$ of DNT. However, analyses performed by sorbing the analyte flow onto Tenax for a 10 minute period (to obtain enough DNT with which to perform analysis) and then analyzing the desorbed products with a GC-ECD system indicated that the actual DNT concentration exiting the tubing and available to be detected was approximately $0.2-0.4 \mathrm{ppb}$.

Eight nominally identical poly(methyloctadecylsiloxane)-carbon black composite detectors were used in this experiment. The detectors were spray-coated onto a ceramic substrate that had pairs of leads spaced $1.0 \mathrm{~mm}$ apart. The leads were 3.5 $\mathrm{mm}$ in length and $0.1 \mathrm{~mm}$ in width and were interdigitated such that the total width contacting a given detector film was 3.0 $\mathrm{mm}$. The outputs of every pair of leads from each detector were connected to a printed circuit board that was equipped with electronics that read the resistance signals to a precision of $<5 \mathrm{ppm}$ every $0.5 \mathrm{~s}$ on the entire bank of detectors. The detectors were placed perpendicular to the output of the DNT flow and were approximately $0.5 \mathrm{~cm}$ from the end of the tubing.

The experimental protocol consisted of one hour of exposure to air only, followed by ten control exposures to $5 \mathrm{~s}$ DNT pulses spaced every $605 \mathrm{~s}$, followed by a randomized sequence of 20 exposures/nonexposures to DNT spaced every $605 \mathrm{~s}$. The data were then analyzed independently without knowledge of the actual order of the randomized sequence of exposures/nonexposures.

A run was also performed to investigate whether responses would be obtained due to small changes in the flow rate of gas to the detectors. For this experiment, the existing lines were unhooked at the outlets of both mass flow controllers (the one feeding the DNT generator and the one providing diluent air). The lines were then replaced with lines and a union- $T$ that bad never been exposed to DNT or to solvents. The lengths of the flow paths with the new lines in place approximated those in the DNT dilution system. A run of four $60 \mathrm{~s}$ exposures, each separated by $10 \mathrm{~min}$, was performed. In this run, $5 \%$ of the air during each exposure came via the mass flow controller that was normally used to feed the DNT generator. The total flow rate at all times was $10 \mathrm{~L}-\mathrm{min}^{-1}$.

\subsection{Dependence of Detector Response on DNT Flow Rate at Constant DNT Concentration}

A separate set of experiments was performed to evaluate the dependence of DNT detection on the flow rate of DNT to the detectors. For this run, a poly(methyloctadecylsiloxane)-carbon black mixture was spray-coated onto the edges of glass slides. Prior to the deposition of the sensor film, conductive coatings bad been deposited onto both surfaces of the slides. Spacers were then placed between these edge-coated slides. The result was a detector with a width of $\approx 6 \mathrm{~mm}$ that had slits $0.13-0.25 \mathrm{~mm}$ in width spanning the length of the detector. This ventilated detector assembly was then cemented into one end of a section of vacuum hose. The other end of the hose was connected to a vacuum pump. A flow meter was placed in the line to monitor the flow rate through the slits in the detector. The rectangular detector face was fitted into a similarlysized aperture in a Teflon block, the fit being loose enough that gas flow onto the detector could escape around the edges. The output tube of the gas mixer was fitted to a second teflon block that was bolted to the block holding the detector assembly, creating a small chamber with a volume of about $0.3 \mathrm{~cm}^{3}$. The resulting distance between the gas mixture outlet and the detector was $\approx 5 \mathrm{~mm}$. The resistance of the detector was measured by connecting the leads to one channel in a data acquisition board that recorded the resistance versus time data. The data were then transferred to a laptop computer. 
Four trials were performed, with each trial using vapor emerging from the DNT-containing analyte tube diluted to $5 \%$ by volume with background air. In experiment 1,10 exposures were made following a $20 \mathrm{~min}$ purge with air at $10 \mathrm{~L}-\mathrm{min}^{-1}$. Each DNT exposure was $10 \mathrm{~s}$ in length. The total flow rates into the detector chamber were varied progressively, starting at $1 \mathrm{~L}-\mathrm{min}^{-1}$ for the first exposure and ending with an exposure at $10 \mathrm{~L}$-min. Each exposure was followed by a purge at $10 \mathrm{~L}$ $\mathrm{min}^{-1}$ of background air. Prior to each exposure, the flow through the vacuum line drawing gas through the detector was set to produce a flow rate that was $1 \mathrm{~L}-\mathrm{min}^{-1}$ less than the flow rate impinging onto the detector chamber. This positive differential flow rate arrangement was used to avoid drawing in ambient air through the remaining gap between the detector and the walls of the chamber.

In experiment 2, 10 exposures were made using the same ascending series of total flow rates into the chamber (i.e. 1-10 L$\min ^{-1}$ ), but no vacuum was applied during any of the exposures.

In experiment 3, the same ascending series of flow rates into the chamber was used, and the same ascending series of vacuum-induced flow rates through the detector as in experiment 1 was employed, but no analyte (DNT) was present.

In experiment 4 , the flow rate of DNT (at $5 \%$ of its vapor pressure at $20^{\circ} \mathrm{C}$ ) into the chamber as not varied, being maintained at $10 \mathrm{~L}-\mathrm{min}^{-1}$ for all 10 exposures. Vacuum-induced flow rates through the detector were, however, varied in the same way as in experiments 1 and 3, beginning with no flow for the first exposure and ending with $9 \mathrm{~L}-\mathrm{min}^{-1}$ during the $10^{\text {th }}$ and final exposure.

\section{RESULTS}

\subsection{Response to $5 \mathrm{~s}$ Pulses of DNT Vapor at Constant Flow Rate}

Figure 1 shows the resistance versus time profile computed by averaging over the bank of eight nominally identical poly(methyloctadecylsiloxane)-carbon black detectors that were placed perpendicular to the outlet of the DNT flow. The red vertical lines show the ground truth of when the DNT puffs were applied. The first ten lines represent the control set. Note that the time axis spans over 6 hours $(22,000 \mathrm{~s})$. The series of "bumps" that are visible on this long time scale plot are not related to the DNT pulses, and in fact represent environmentally-induced oscillations in the baseline resistance of the detector. The DNT-induced behavior occurs on a 5 second time scale that is not discernible on this plot.

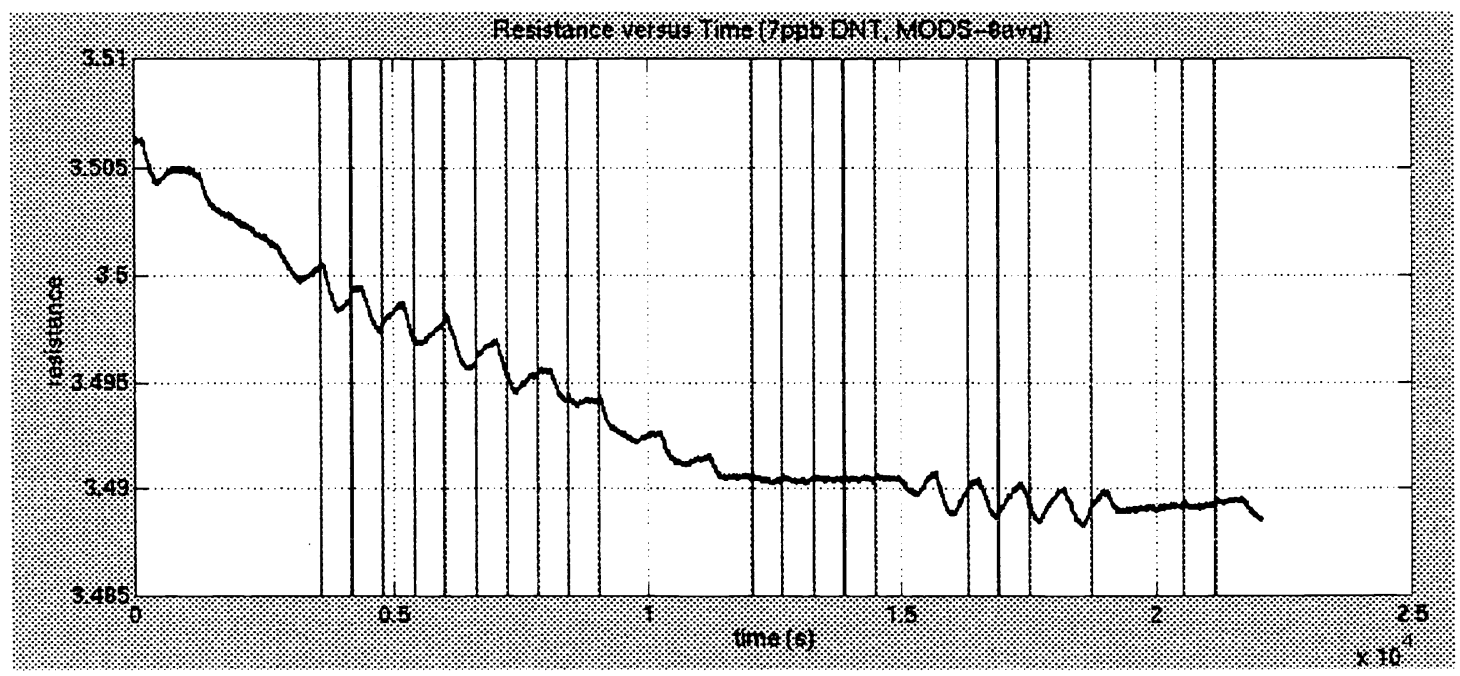

Figure 1: Resistance versus time profile computed by averaging over the bank of eight nominally identical poly(methyloctadecylsiloxane)-carbon black composite detectors. (The $\mathrm{y}$-axis is in units of $10 \mathrm{k} \Omega$.)

Using essentially a matched filter algorithm with adaptive background subtraction, all DNT exposures and non-exposures were correctly identified within the randomized sequence. The black circles show local maxima of the detector output that 
exceeded a given threshold. Based on this selection criterion, all of the DNT exposures were detected with no false alarms. In fact, a much stronger result was obtained: the separability at the detector output was sufficient for all DNT exposures (in the control set and the randomized set) to be correctly identified with zero false alarms over the entire $>6$ hour duration of the experiment.

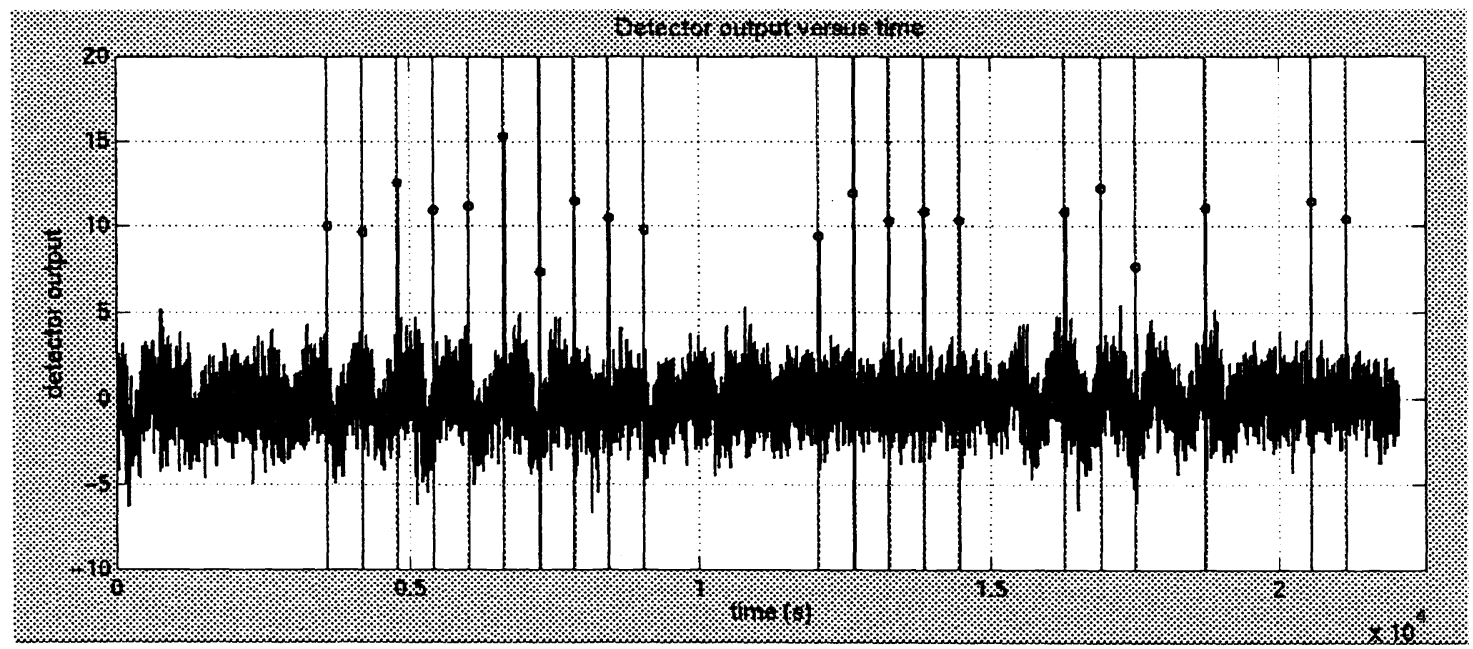

Figure 2: Detector output versus time over the 6+ hours of the experiment. The vertical lines show the ground truth of when DNT puffs were applied. The units on the $y$-axis are in standard deviations of the signal relative to the local background of the detector.

The highest detector output value (15.3) occurred at the 6th control sample. The resistance versus time profile for this sample is shown in detail in Figure 3a. The detector output versus time is shown in Figure $3 b$.
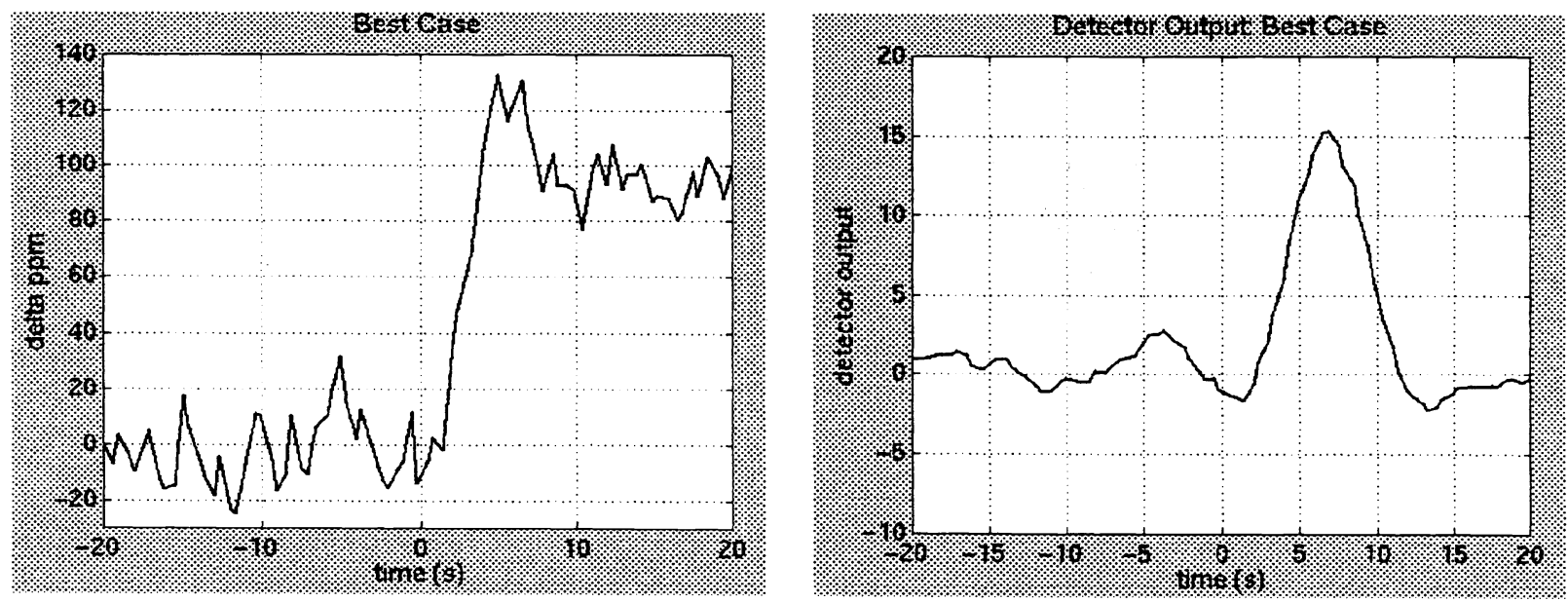

Figure 3: (a) Resistance (delta ppm) behavior of the DNT exposure that produced the largest detector output value (control sample 6). (b) Detector output value (unitless) versus time.

An intermediate case (roughly the median in detector output fidelity) occurred at the 4th control sample. The resistance versus time profile for this sample is shown in detail in Figure $4 \mathrm{a}$. The detector output versus time is shown in Figure $4 \mathrm{~b}$. 

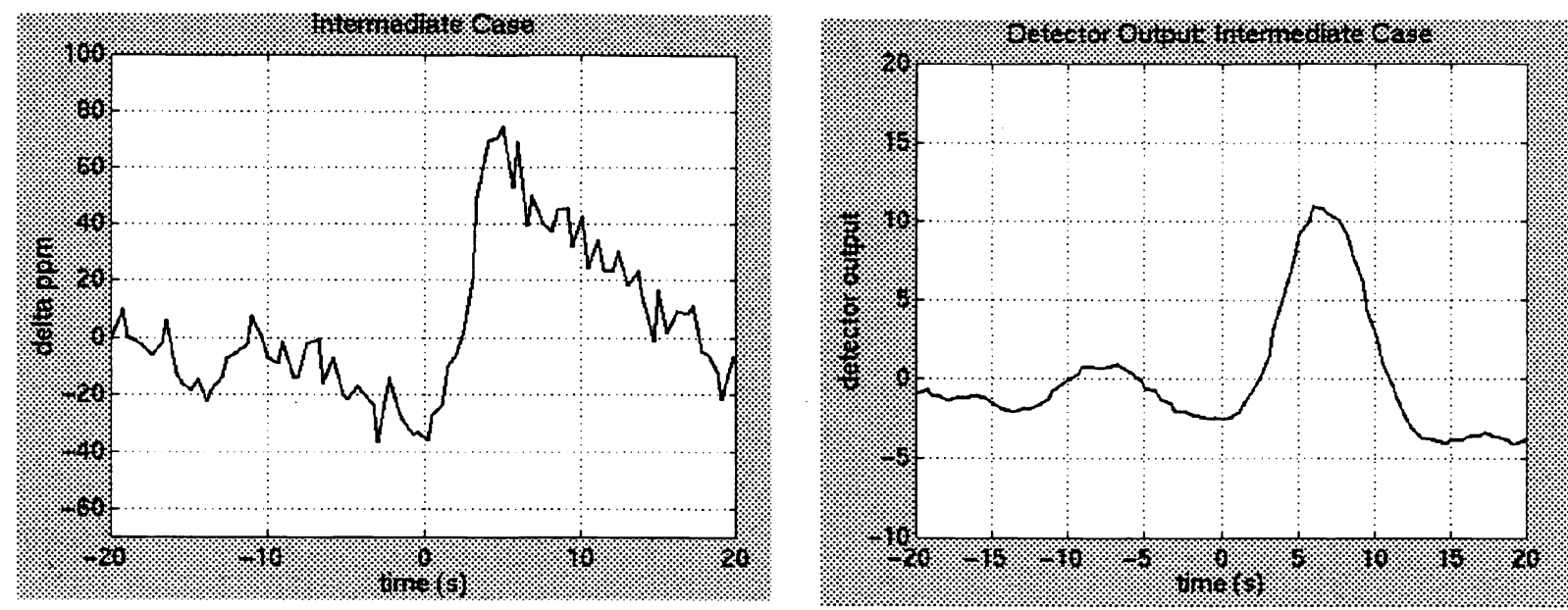

Figure 4: (a) Resistance (delta ppm) behavior of the DNT exposure that produced an intermediate detector output value (control sample 4). (b) Detector output value (unitless) versus time.

The lowest detector output value (7.35) occurred at the 7th control sample. The resistance versus time profile for this sample is shown in detail in Figure 5a. The detector output versus time is shown in Figure $5 b$.
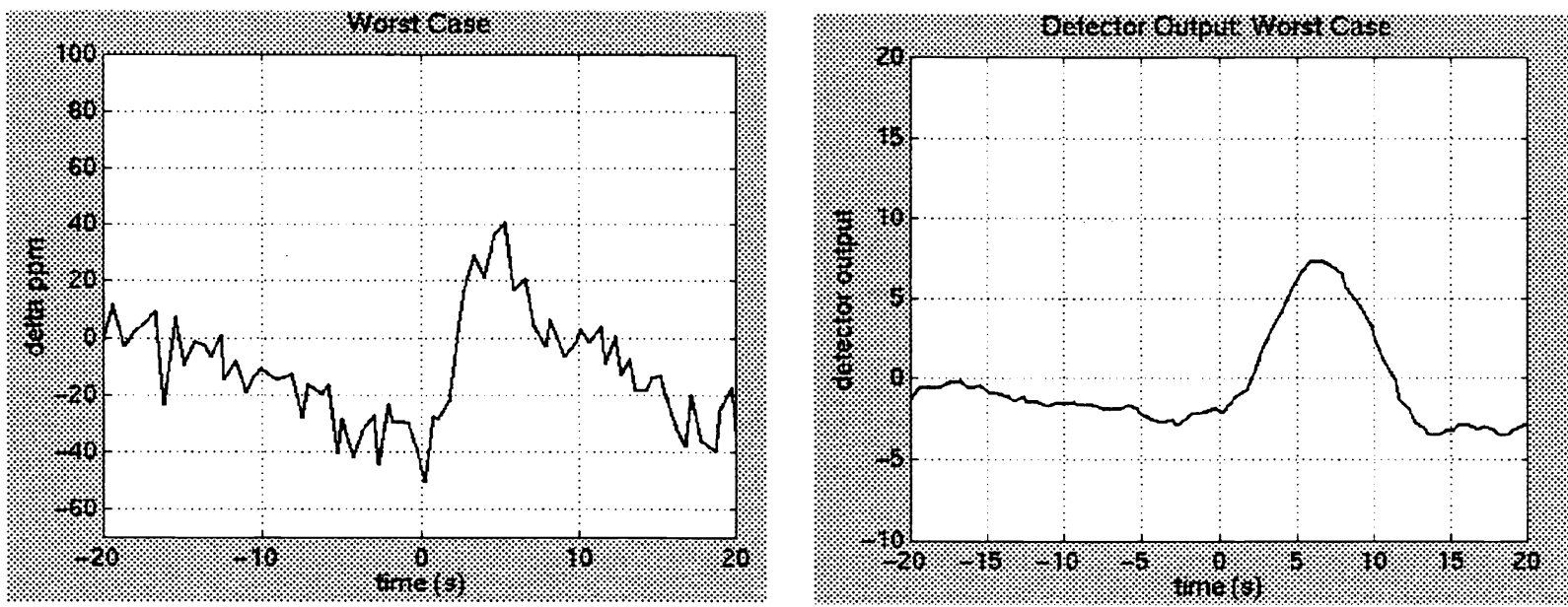

Figure 5: (a) Resistance (delta ppm) behavior of the DNT exposure that produced the smallest detector output value (control sample 7). (b) Detector output value (unitless) versus time.

Although all the DNT exposures were perfectly separated from the background with zero false alarms, it is interesting to look at the "close calls" or "near false alarms". In Figure 2 there are 4 places where the detector output on the background exceeds a threshold of 5 but is still well below the minimum target value of 7.35. The detailed resistance versus time profiles for these 4 "near false alarms" are shown in Figure 6a-d. 

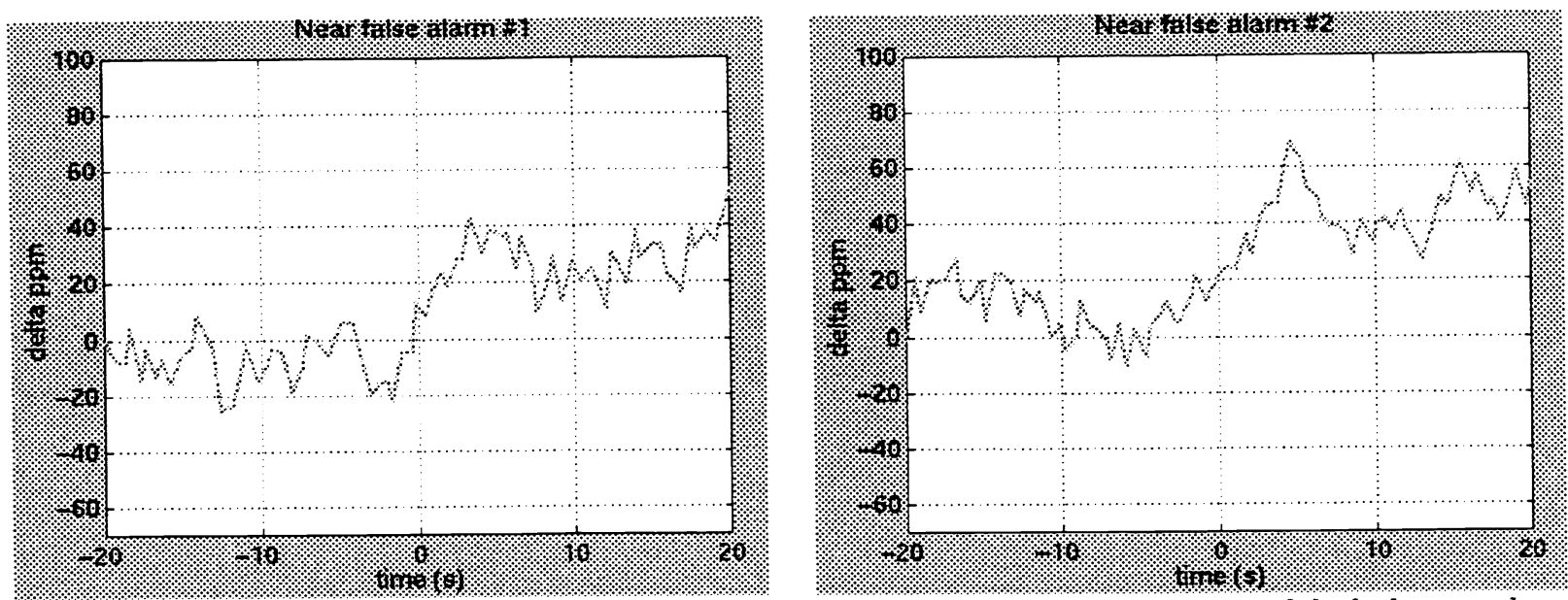

Figure 6: Resistance (delta ppm) behavior in the four background windows that slightly above 5, while the lowest value produced the largest detector output values. The maximum detector output produced over these background samples was produced for a real DNT exposure was 7.35 .

\subsection{Dependence of Detector Response on DNT Flow Rate at Constant DNT Concentration}

Because the vapor pressure of DNT is so low, the detectors are operating far from their equilibrium response values. In this situation, increasing the mass of DNT that impinges upon the detector in a given time period will produce an increase in the measured resistance response. A set of experiments was performed in order to evaluate whether there was an experimentally accessible flow rate that provided saturation of the detector, or whether improved signals could be obtained at increased flow rates up to the available limit in our laboratory system.

These experiments were performed as described in the experimental section above, with the flow rate impinging upon the detector assembly slightly exceeding the flow rate being pulled through the ventilated detector assembly. This simulated conditions of a "real world" sampling unit that would actively transport analyte vapor to the detectors. As indicated in Figure 7 , pulling analyte through the detector at a rate about $1 \mathrm{~L}-\mathrm{min}^{-1}$ less than the flow rate of gas into the chamber generally resulted in an increase in detector response of a factor of 2 . This was particularly noticeable at higher flow rates.
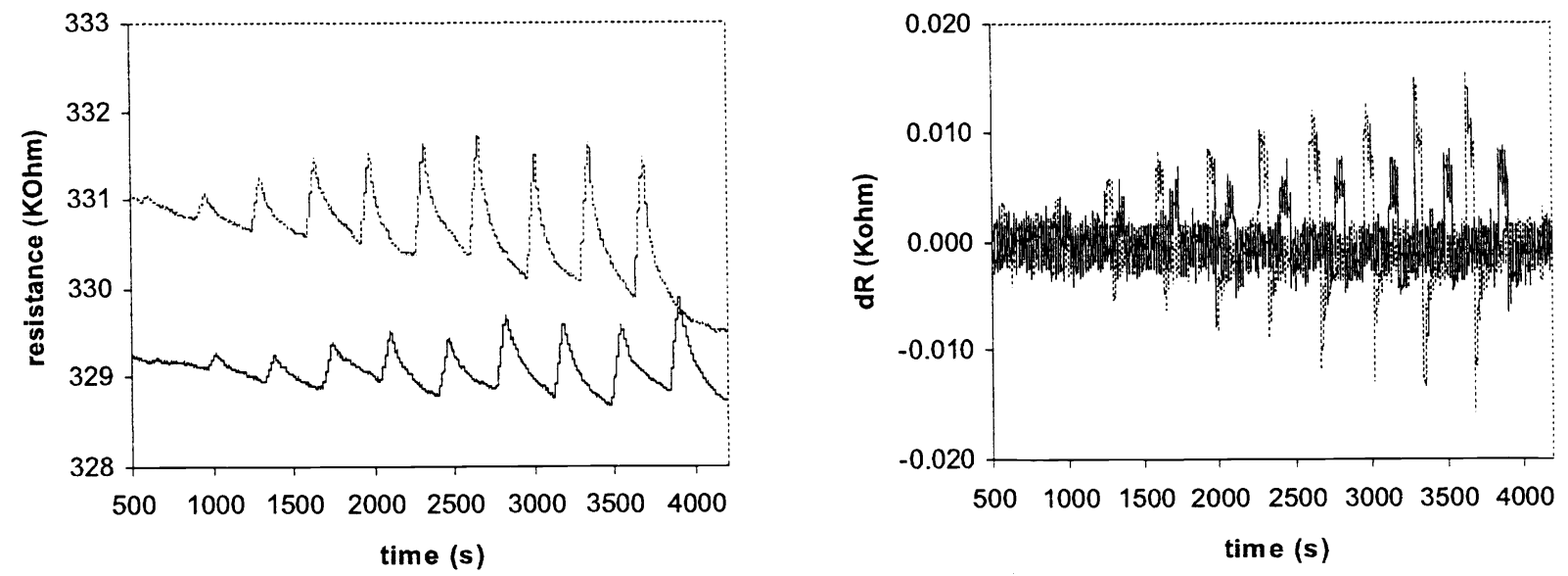

Figure 7: Dependence of signal from a ventilated sensor on flow rate where the total flow rate through the chamber ranged from 1 to $10 \mathrm{~L}-\mathrm{min}^{-1}$. (a) Resistance transients for 1 minute exposure to DNT at 5\% of its vapor pressure (top: vacuum on; bottom vacuum off). (b) Change in resistance as a function of time indicating magnitude of slope during exposure. 
When flow into the detector chamber was kept at a constant, high rate $\left(10 \mathrm{~L}-\mathrm{min}^{-1}\right)$, the detector response increased, apparently due to increased flow through the detector slits. These data clearly imply a critical role for sampling design in achieving rapid and high sensitivity detection of DNT and other low vapor pressure analytes.
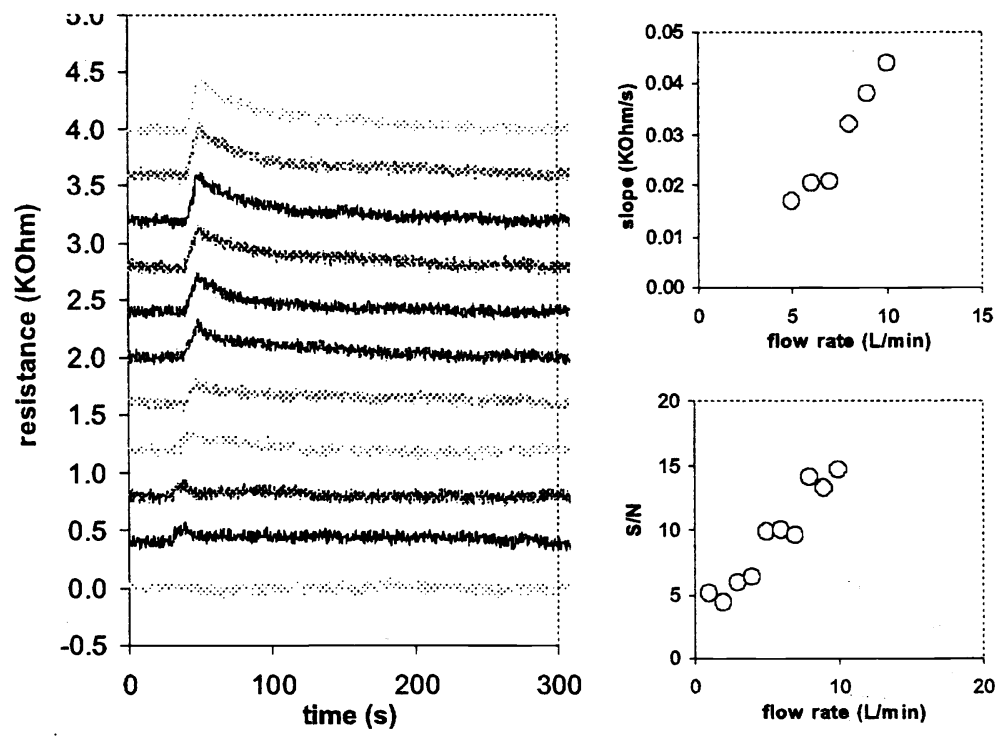

Figure 8: Ventilated sensor response characteristics for $5 \mathrm{~s}$ exposures to $10 \mathrm{~L}_{\text {-min }}^{-1}$ total flow rates of $5 \%$ DNT as a function of flow rate through the sensor. (a) Response transients ranging from no flow through the sensor (second from the bottom) to 9 L-min-1 through the sensor (top). (b) Response slope as a function of flow rate through the sensor. (c) Signal to noise after $5 \mathrm{~s}$ exposure as a function of flow rate.

analytes.

\subsection{Sampler Design}

In response to these results, AeroVironment, Inc. designed and fabricated several prototype circuit board detector concepts (Figure 9) and a detector test-fixture (Figure 10).
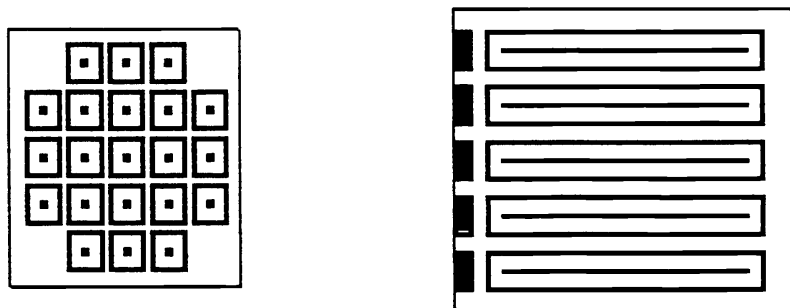

Figure 9. Detector circuit boards. Left: 21-element "flow-through" detector. Right: High-low vapor pressure stacking detector.

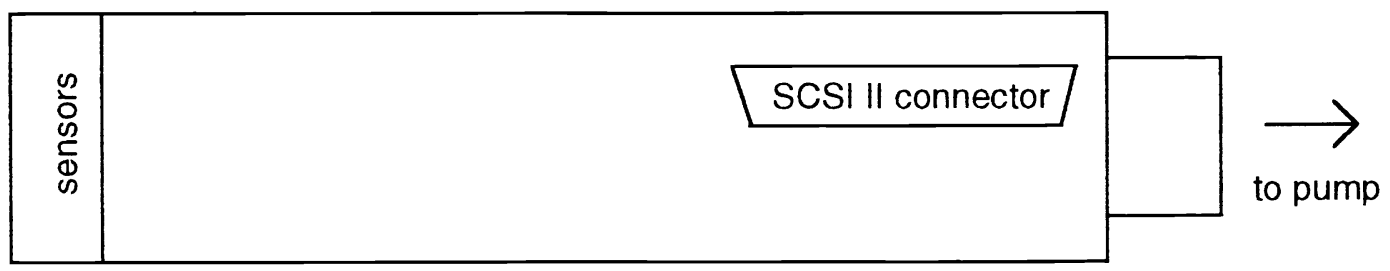

Figure 10. Detector head test fixture for flow-through detector. 
The detectors of Figure $9 \mathrm{~b}$ are stacked so that their leading edges are normal to the flow, so in essence the linear gaps between detector plates of the Figure 9 design are a 1-dimensional analog of the detector hole flow path in the design of Figure 9a. Both of these have their advantages and disadvantages, but they should perform quite similarly to each other based on the results of simulations of the flow and analyte capture properties of these systems.

The design principles of these detector heads are summarized in the remainder of this section.

The smallest allowable detector open (through-flow) area is dictated by the specified flow rate, $\dot{V}$, and the speed of sound in air, $a$. Using representative numbers, the airflow open area must be on the order of,

$$
\ell^{2} \sim \frac{\dot{V}}{a M}=\frac{10 \mathrm{~cm}^{3} / \mathrm{s}}{(34000 \mathrm{~cm} / \mathrm{s})(0.6)} \sim(0.2 \mathrm{~mm})^{2}
$$

where $M$ is the Mach number, or fraction of sound speed in the holes. Note that sonic flow through the holes can usually be achieved by pulling a vacuum on the backside of the perforated plate.

Another consideration is that the optimum signal to noise requirements suggest a detector area, $A_{p}$, of,

$$
A_{p}=\frac{\varepsilon}{f} \frac{1}{K} \frac{V_{a}}{t_{p}} \sim \frac{0.5}{0.25} \frac{1}{10^{8}-10^{9}} \frac{10 \mathrm{~cm}^{3}}{10^{-5} \mathrm{~cm}} \sim(1.4 \mathrm{~mm})^{2} \diamond(0.4 \mathrm{~mm})^{2}
$$

where $V_{a}$ is analyte air volume, $t_{p}$ is the detector polymer thickness, $\varepsilon$ is the collection efficiency, and $f$ is the analyte fraction of saturation. This occurs because larger detector areas will simply produce a dilution of the available DNT into larger detector volumes, thereby producing less resistance change in such detector films. Because the signal scales linearly with the concentration of sorbed analyte, whereas the noise scales like the square root of the detector film area (for constant film thickness), this favors smaller detector areas, as indicated in eqn (2).

The analysis above suggests that use of a flow-through detector configuration of roughly $2-25 \%$ open area (98\%-75\% solidity, with the exact value depending on the assumed partition coefficient of DNT into the polymer film) would be optimal for DNT detection.

Simulations of detectors having $1 \%$ open area suggest that the capture effectiveness of the perforated plate arrangement scales with the flow Reynolds number. However, the capture effectiveness can be bounded from below by $50 \%$ (the value used in Equation 2 above) for Reynolds numbers up to 100 which, for the current exercise, is the limiting case of a detector with one or two holes and an open area of $1-2 \%$. It is probably sufficient to have enough holes to ensure even flow into the detector.

Significant improvements over this design (up to $\sim 90 \%$ analyte capture) can be expected when the Reynolds number is on the order of 1 (very many small holes $\sim 1 \mu \mathrm{m}$ in diameter). Micro-machining methods are required to satisfy the dimensions of Equation (2) above. However, a 10x version of the flow-through detector concept is achievable using standard circuit and flex-board processes. Having a length scale 10 times larger than optimal, these can be expected

to have roughly $1 / 10$ the signal to noise of the example above

These designs described above take advantage of our understanding to date of how to best accomplish several key features simultaneously:

1) they insure that the low vapor pressure material in the analyte puff being sampled encounters a minimum volume of detector film, consistent with the volume of detector needed based on the equilibrium partition coefficient data obtained previously for the particular films) to sorb all of the DNT in this analyte plug into the detector film; 
2) they insure that the maximum quantity of analyte impinges upon and can be sorbed by the film, leaving only clean air to pass through the holes/slots when encountering a puff of DNT-containing analyte approximately $10 \mathrm{~cm}^{3}$ in volume;

3) the design of figure 9 also allows sorption of high vapor pressure analytes where they will have the greatest signal/noise, along the faces of the detectors. This not only allows differential removal of high vapor pressure analyte signals but also insures that the low vapor pressure analytes are captured where their $\mathrm{S} / \mathrm{N}$ ratio is the highest and the high vapor pressure analytes are captured where their $\mathrm{S} / \mathrm{N}$ ratio is the highest.

These designs are based on the validated performance of simple mockups and in dye tests, in conjunction with DNT flow rate vs sensitivity tests of mock-ups in analogous designs. These designs are also supported by both analytical flow dynamics solutions and by CFD simulations. The hole (or gap width in the 1-D case) diameter is bigger than one would ultimately wish to fabricate, but these designs should provide a very good rapid prototype test bed for assessing the performance of the detector/sampling system in real-world laboratory and field environments.

The above samplers are being tested with a single polymer to verify their predicted performance. Next we will make arrays of detectors instead of a single detector film covering all of the available sensing area. The improved fluidics and sampler design will produce the most mass on the least detector area, maximizing the $\mathrm{S} / \mathrm{N}$ presumably values below the ppb level. They will also allow for improved rejection of signals due to high vapor pressure analytes, which are not well-detected at the small area detectors. In addition, these systems are designed to operate optimally at flow rates that correspond to sampling $1-10 \mathrm{~cm}^{3}$ of analyte per second, which is in accord with estimates of the volume desired to be sampled spatiotemporally above a land mine in order to perform efficient area searches by a walking deminer.

\section{ACKNOWLEDGMENTS}

We acknowledge an OSD ARO MURI for demining, and DARPA, for support of this work.

\section{REFERENCES}

(1) Norman, R. H. Conductive Rubbers and Plastics; Elsevier: Amsterdam, 1970.

(2) Carbon Black-Polymer Composites; Sichel, E. K., Ed.; Marcel Dekker, Inc.: New York, 1982.

(3) Medalia, A. I. Rubber Chem. Tech. 1986, 59, 432.

(4) Ford, C. J. In U.S. Patent 2,691,134, 1951.

(5) Newton, R. G. J. Rubber Res. 1946, 15, 35

(6) Sands, A. G.; McDowell, M. V. Rubber Age, New York 1956, 80, 500.

(7) Boyd, J.; Bulgin, D. J. Text. Inst. Proc. 1957, 48, 66.

(8) Lundberg, B.; Sundqvist, B. J. Appl. Phys. 1986, 60, 1074.

(9) Ruschau, G. R.; Newnham, R. E.; Runt, J.; Smith, B. E. Sens. Actuators 1989, $20,269$.

(10) Talik, P.; Zabkowskawaclawek, M.; Waclawek, W. J. Mater. Sci. 1992, 27, 6807.

(11) George, V.; Jenkins, T. F.; Leggett, D. C.; Cragin, J. H.; Phelan, J. M.; Oxley, J. C.; Pennington, J. Proc. SPIE 1999, 3710, 258

(12) Cummings, C., private communication.

(13) Severin, E. J.; Doleman, B. J.; Lewis, N.S. Anal. Chem.; 2000; 72; 658 